\title{
Nebulized lipid nanoparticles
}

\begin{tabular}{|c|c|}
\hline $\begin{array}{l}\text { To identify lipid } \\
\text { nanoparticle } \\
\text { design princi- } \\
\text { ples for lung } \\
\text { delivery, the } \\
\text { researchers } \\
\text { developed } \\
\text { an in vivo } \\
\text { cluster-based } \\
\text { iterative } \\
\text { screening } \\
\text { approach }\end{array}$ & $\begin{array}{l}\text { Lipid nanoparticles have enabled the } \\
\text { systemic delivery of nucleic acids, for } \\
\text { example, COVID-19 mRNA vaccines. } \\
\text { However, many diseases, including } \\
\text { conditions caused by respiratory } \\
\text { viruses, would greatly benefit from } \\
\text { drug delivery directly into the lungs. } \\
\text { Now, writing in Nature Biomedical } \\
\text { Engineering, Philip J. Santangelo, } \\
\text { James E. Dahlman and colleagues } \\
\text { developed an in vivo workflow for } \\
\text { the design and optimization of lipid } \\
\text { nanoparticles to efficiently deliver } \\
\text { mRNA to the lungs via nebulization. } \\
\text { The road to optimizing lipid } \\
\text { nanoparticles for systemic nucleic } \\
\text { acid administration has been long } \\
\text { and winding, and was based on } \\
\text { extensive in vitro screening of lipid } \\
\text { nanoparticles with different chemical } \\
\text { designs, of which only very few made } \\
\text { it to preclinical and clinical stages, } \\
\text { in part because in vitro nanoparticle } \\
\text { delivery may not be a good predictor } \\
\text { of in vivo nanoparticle delivery. } \\
\text { Nanoparticle-based formulations } \\
\text { have also been explored for nucleic }\end{array}$ \\
\hline
\end{tabular}

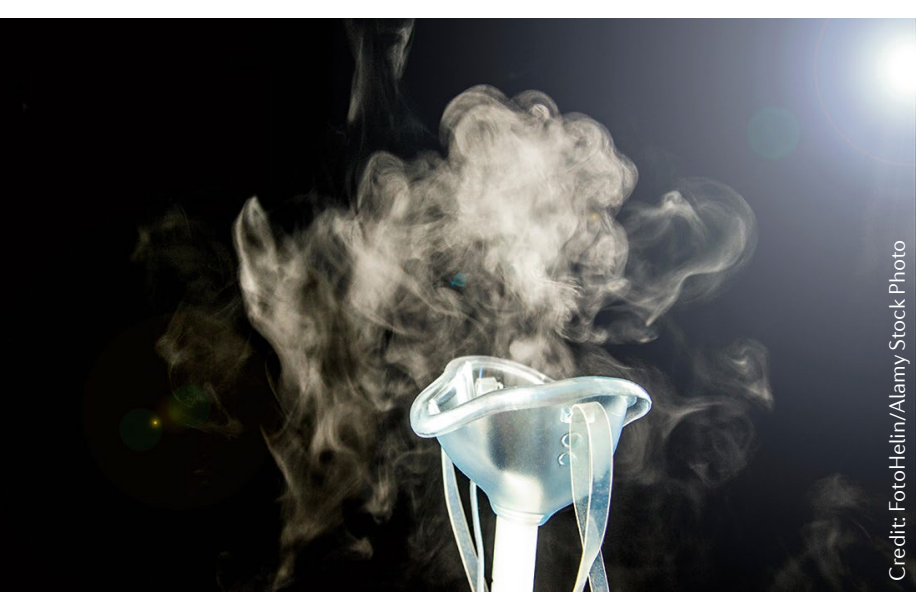

acid delivery into the lungs but have not yet achieved therapeutic levels of expression. This may be related to the fact that lipid nanoparticle design faces distinct challenges when it comes to delivery via nebulization and inhalation.

"The mRNA-nanoparticle formulation first has to survive the nebulizer, that is, shear forces that can disrupt its structure, and then has to be taken up efficiently by the epithelium without inducing inflammation," explains Santangelo. "These challenges are different from those related to systemic delivery, which requires the nanoparticle to interact with serum proteins, avoid circulating immune cells, avoid liver clearance, and extravasate into the right tissue type."

To identify lipid nanoparticle design principles for lung delivery, the researchers developed an in vivo cluster-based iterative screening approach. The workflow is based on defining distinct chemical traits, each treated as an axis in an $\mathrm{N}$-dimensional chemical space, including the amount of polyethylene glycol (PEG) added to the lipid nanoparticle, the structure of the lipid-PEG molecule, the charge of the phospholipid, and the presence or absence of cholesterol. In this design space, six extreme groups are then defined by varying molar ratios, components and charge, and 8-12 lipid nanoparticles are formulated for each group, all based on the oligomer-lipid conjugate $7 \mathrm{C} 1$. These nanoparticles can then be simultaneously tested for mRNA delivery via nebulization in vivo.
Using this workflow, the authors found that PEG-lipid molecules, but not cholesterol and helper lipids, are crucial to forming stable 7C1-based lipid nanoparticles. Moreover, a combination of cationic helper lipids and high-molar percentages of PEG increases mRNA delivery after nebulization, suggesting that nanoparticle chemistry plays a more important role than particle size in nebulized delivery.

Based on these design rules, a new nanoparticle with a high amount of PEG-lipid and cationic helper lipid was designed for lung delivery, termed nebulized lung delivery 1 (NLD1). NLD1 can efficiently deliver mRNA encoding the therapeutic antibody FI6 that binds haemagglutinin to neutralize various subtypes of influenza A viruses. Indeed, pre-treatment with NLD1 via nebulization rescued the life of mice inoculated with the influenza virus $\mathrm{H} 1 \mathrm{~N} 1$.

The researchers are now also evaluating species compatibility, beyond mice, and explore other mRNA cargos to assess applicability of NLD1 to other lung diseases. Moreover, they would like to further improve delivery efficiency. "Using these direct in vivo screens, we have already found NLD2, a second-generation nebulized lipid nanoparticle, which outperforms NLD1 in terms of delivery efficiency," says Dahlman.

Christine Horejs ORIGINAL ARTICLE Lokugamage, M. P. et al. Optimization of lipid nanoparticles for the delivery of nebulized therapeutic mRNA to the lungs. Nat. Biomed. Eng. 5, 1059-1068 (2021) 